\title{
Transversely modulated wave packet
}

\author{
V. N. Salomatov \\ Irkutsk State Transport University \\ 15 Tchernyshevsky st., 664074 Irkutsk, Russia
}

E-mail: sav@irgups.ru

\section{Abstract}

The wave packet consisting of two harmonic plane waves with the same frequencies, but with different wave vectors is considered. The dispersion relation of a packet is structurally similar to the dispersion relation of a relativistic particle with a nonzero rest mass. The possibility of controlling the group velocity of a quasi-monochromatic wave packet by varying the angle between the wave vectors of its constituent waves is discussed.

Key words: quasi-monochromatic waves; group velocity; dispersion relation; longitudinal modulation; coherence time.

The widespread practical use of wave packets in microwave technology, as well as in the technique of the optical range, in particular in the technique of ultrashort laser pulses [1-9], necessitates a detailed study of specific wave packets.

Noteworthy is a packet consisting of two quasi-monochromatic plane waves propagating at an angle to each other. Despite the obvious simplicity of such a packet, its properties, in particular, the dependence of its group velocity on the angle between the components, have not been studied in detail.

Let's consider two harmonic plane waves propagating at an angle $\vartheta$ to each other

$$
\begin{aligned}
& \mathrm{a}_{1}=\operatorname{Acos}\left(\mathbf{K}_{\mathbf{1}} \mathbf{r}-\omega \mathrm{t}\right), \\
& \mathrm{a}_{2}=\operatorname{Acos}\left(\mathbf{K}_{2} \mathbf{r}-\omega \mathrm{t}\right) .
\end{aligned}
$$

Here, $\mathrm{A}$ is the amplitude, $\omega$ is the cyclic frequency, $\mathbf{K}_{\mathbf{1}}$ and $\mathbf{K}_{\mathbf{2}}$ are wave vectors, moreover, $\mathbf{K}_{\mathbf{1}}=\mathbf{k}+\mathbf{q}, \mathbf{K}_{\mathbf{2}}=\mathbf{k}-\mathbf{q}$. The vector $\mathbf{q}$ is directed perpendicular to the vector $\mathbf{k}$ (Fig. 1).

Waves (1) and (2) satisfy the linear wave equation. Linear combinations of solutions also satisfy it, including

$$
\mathrm{a}=\mathrm{a}_{1}+\mathrm{a}_{2}=\mathrm{A}\{\cos [(\mathbf{k}+\mathbf{q}) \mathbf{r}-\omega \mathrm{t}]+\cos [(\mathbf{k}-\mathbf{q}) \mathbf{r}-\omega \mathrm{t}]\}=
$$




$$
=2 A \cos \frac{\mathbf{k}+\mathbf{q}-\mathbf{k}+\mathbf{q}}{2} \cos \frac{2 \mathbf{k r}-\mathbf{2 \omega t}}{2}=2 A \cos q r \cdot \cos (\mathbf{k r}-\omega \mathrm{t})=\mathrm{a}_{\mathrm{q}} \mathrm{a}_{\mathrm{k}}
$$

Here

$$
\begin{aligned}
& \mathrm{a}_{\mathbf{q}}=2 \cos \mathbf{q} \mathbf{}, \\
& \mathrm{a}_{\mathbf{k}}=A \cos (\mathbf{k r}-\omega \mathrm{t}) .
\end{aligned}
$$

For simplicity, we assume that (1-3) describe waves, with a1, a2, and a varying in directions perpendicular to the plane of the figure.

Note that in $(1-3) \omega \neq \mathbf{c k}$, but

$$
\omega=c\left(\mathbf{q}^{2}+\mathbf{k}^{2}\right)^{1 / 2}
$$

This relation may be considered as the dispersion relation for the wave packet (3).

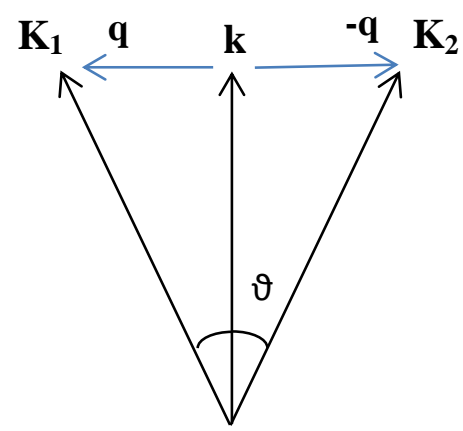

Fig. 1. The directions of the wave vectors of the considered waves.

As is well known $[1,4,5]$, the dispersion relation determines the basic properties of a wave packet, including its group velocity. Let's pay attention to the fact that in the case under consideration the dispersion relation (6) is structurally similar to the dispersion relation for a relativistic particle (e.g., [10-12]), having a rest mass.

Thus, the superposition of waves (1) and (2) leads to the formation of a transversely modulated wave packet (3) with a group velocity

$$
\mathbf{v}=\frac{\partial \omega}{\partial \mathbf{k}}=\mathrm{c} \frac{\mathbf{k}}{\left.\sqrt{\left(\mathbf{q}^{2}\right.}+\mathbf{k}^{2}\right)}=\mathrm{c} \cdot \cos \frac{\vartheta}{2} \text {. }
$$

For the practical use of specific wave packets it is necessary to control their group velocity [13-19].

It can be seen from formula (7) that the group velocity of the considered wave 
packet can be controlled by varying the angle $\vartheta$ between the wave vectors of its constituent waves.

The considered idealized monochromatic wave packet has only the transverse modulation described by the factor (4). As is well known, the monochromaticity of the waves, including the monochromaticity of the considered wave packet, is only a convenient idealization. Real waves have a variation in frequencies, that is, are quasi-monochromatic. This leads to the presence of longitudinal modulation of wave packets [1-9].

The nonmonochromaticity of the considered wave packet can be created artificially in accordance with a change in the transmitted signal. It is of interest to investigate experimentally the possibility of controlling the group velocity of a packet by changing the angle between the wave vectors of its constituent waves within the coherence time.

\section{References}

[1] Thiele C. 2006 Wave Packet Analysis, CBMS Regional Conference Series in Mathematics, v.105.

[2] Greenfield E., Segev M., Walasik W. \& Raz O. 2011 Phys. Rev. Lett. 106, 213902.

[3] Voloch-Bloch N., Lereah Y., Lilach Y., Gover A. \& Arie A. 2013 Nature 494, 331.

[4] Tzortzakis S. 2015 Linear and nonlinear exotic light wave packets physics and applications, in Frontiers in Optics, OSA, paper LM3I.4.

[5] Hu Y., Li Z., Wetzel B., Morandotti R., Chen Z., Xu J. 2017 Scientific Reports, 7 (8695). ISSN 2045-2322.

[6] Shalaev V.M. 2007 Nature Photonics. 1., 41.

[7] Froehly L. et al. 2011 Opt. Express 19, 16455.

[8] Rulliere C. (ED.) 2005 Femtosecond Laser Pulses, Springer, Berlin.

[9] Zhu Y. et al. 2011 Cent. Eur. J. Phys. 9(4), 956.

[10] ] F. Dyson F. 2011 Advanced Quantum Mechanics. World Scientific, Singapore.

[11] Salomatov V.N. 2017 Phys. Essays 30, 177. 
[12] Salomatov V.N. 2018 Phys. Essays 31, 164.

[13] Lukin M.D., Imamoglu A. 2001 Nature, 413, 273.

[14] Yanik M.F. et al., Patent US 7269313 B2.

[15] Terrel M.A.et al., Patent US 2011/0134432 Al.

[16] Ham B., Patent US 2010/0232792 Al.

[17] Gan Q.et al., Patent US 2010/0110525 Al.

[18] Jiang T. et al, 2009 Optic Express, 17, (1), 170.

[19] Savo S. et al 2011Appl. Phys. Lett. 98 ,17. 